\title{
Making the case for Community Interpreting in health care: from needs assessment to risk management
}

\author{
Diana Abraham \\ Faculty of Environmental Studies \\ York University \\ Marco A. Fiola \\ Department of French and Spanish \\ Ryerson University
}

In Canada, community interpreting is little recognized and valued by public institutions, including those in the healthcare sector. Although many healthcare practitioners recognize the crucial role played by interpreters in delivering healthcare services, some of them ascribe to the notion that the inability to communicate with English-speaking or French-speaking patients is the patient's problem, and that any linguistic miscommunication which may occur is the responsibility of the patient. This attitude contributes to the degree to which healthcare practitioners rely on interpreting provided by family members, including children, without consideration either for risks of errors and omission or for potential violations of confidentiality, which are likely to occur when asking friends or relatives to provide interpreting services. This "wall of resistance" has been deemed responsible for much of the difficulty experienced in Canada by immigrant and minority language advocacy groups in trying to ensure community interpreting services for immigrants, refugees and those Canadians with limited proficiency in English and/or French. A recently completed research study funded by the Government of Canada suggests that a paradigm shift may be operating in the healthcare sector, and that instead of still seeing language barriers solely as a human rights issue, language barriers should be considered from a risk-management perspective as well. This paper will review some of the main findings of this study.

\section{Introduction}

In 2003, the Healthcare Interpretation Network (HIN) of Toronto, in collaboration with Critical Link Canada, received funding from Health Canada to conduct research aimed at assessing the language barriers to primary healthcare $^{1}$ within the Canadian public health system. The overall objectives of this project were to investigate the current approaches used by primary health care providers in delivering health care when they do not share a common language with the patient, and the models of service delivery utilized in the delivery of particular sectors of primary health care. An anticipated outcome of the project was the identification of systemic approaches 
to addressing the language barriers existing within the primary healthcare sector.

The project included a review of Canadian and selected international literature and an investigation into the current state of primary healthcare service delivery to patient populations with limited-English or limitedFrench proficiency (LEP/LFP) in the three largest Canadian cities - Montreal, Quebec; Toronto, Ontario and Vancouver, British Columbia. These sites, which are the main points of entry for newcomers to Canada, were chosen both for the diversity of their immigrant and refugee populations, and the differences in the approaches to the delivery of interpreting services in the healthcare sectors. For example, in Montreal, the Agence de la santé et des services sociaux de Montréal is funded by the province of Quebec to respond to the demand for interpreting and translation services by the healthcare sector in that city. In Vancouver, the Provincial Language Service offers interpreting, translation, cultural competency training and consulting services to support healthcare professionals in ensuring ethno-cultural communities full access to quality healthcare services that are culturally and linguistically appropriate. Toronto has a mix of different approaches, but no systematic mechanism is in place.

The primary objective of the research component of the project, which is the subject of this paper, was to document the current state in the provision of primary healthcare services to patient populations who do not share a common language with the health care providers. It aimed at identifying and assessing current models of practice. This study, which was completed in 2004 (Hoen, Nielsen \& Sasso, 2006), was useful in documenting what was long suspected: practices are not standardized across the country, and there are but a few instances where health care providers have been able to implement systematic mechanisms to ensure that the quality of health care is not hindered by language barriers. It also highlighted the degree to which the delivery of primary care with the assistance of trained health care interpreters is far from generalized.

This paper will review some of the main findings of this study, and will attempt to explain how a paradigm shift may be the key to integrating community interpreters within the primary healthcare service delivery matrix.

After a description of the research methodology employed to interview service providers in the primary healthcare sector, and of some of the research findings, our analysis of the findings will be framed within the contexts of

- $\quad$ The risks involved when working with untrained interpreters;

- the resistance to working with professional interpreters;

- $\quad$ the paradigm shift. 


\section{Research methodology}

A qualitative emergent approach was used in interviews with individuals and in focus groups (see 1.2 Sample) with healthcare interpreters and interpreter service agencies, healthcare practitioners in community-based and hospital settings and immigrant settlement workers. The emergent approach was seen to allow a greater level of flexibility in comparison to inductive or deductive methods. Given the exploratory nature of the research project, it was thought that in order to avoid any interference due to the researchers' preconceptions, this approach would enable informants to delve further into issues that might have been overlooked by researchers in the planning phase of the research. This data-gathering method was also deemed to be suitable for the wide range of informants with varying levels of familiarity with community interpreting in the primary healthcare system.

A questionnaire outline was developed by the researchers for all focus groups and individual informants, asking participants to express their points of view on a wide number of issues relating to, for example, language accessibility to primary health care: the role of the interpreter, the qualities of the ideal interpreter, the availability of trained versus untrained interpreters, and the impact of language barriers on health outcomes.

In the focus group sessions, the interviewer would initiate the discussion by asking a question, after which the informants would be invited to share their views on that question and on each other's comments. In individual interviews, there was no discussion of the views expressed by the informant. All data were audio-recorded.

\subsection{Limitations of the research method}

In this research method, informants have the freedom to raise issues of concern to them; as a result, potential researcher bias is reduced. However, in using this method, the researchers could not be confident of collecting comprehensive information because particular issues may not have been raised by informants in the interviews.

Informants were identified by research advisory committees set up in Vancouver, Toronto and Montreal, then invited to take part in a focus group or to be interviewed individually. In selecting participants, advisory committees aimed to achieve maximum representation of all stakeholder groups. One of the limitations of this sampling method is that informants were selected based on their roles within the primary healthcare system, not on their previous knowledge of the issues related to language accessibility in primary health care.

In weighing the benefits and limitations of the research method, the researchers felt that the emergent research approach provided them with a better tool to achieve the objectives of the research. However, given the wide range of data that were collected, other researchers might be well ad- 
vised to provide clearer directions to informants during the interview process.

Overall, the main limitations of this emergent qualitative research method are (1) the difficulty of arriving at a precisely quantified analysis of the data; and (2) the risk of not addressing all of the intended research questions identified by researchers prior to the actual discussions and interviews.

\subsection{Sample}

A total of 140 individuals from Vancouver, Montreal and Toronto were interviewed, either in focus groups or individually. These informants, who were all from the primary healthcare sector, were members of the following stakeholder constituencies:

- community health centres;

- healthcare interpreters;

- hospital emergency services;

- interpreter service agencies;

- interpreting/translation professional associations;

- nurses;

- physicians;

- $\quad$ settlement workers;

- social workers.

While the hospital emergency services do not fit within the Canadian Government's definition of primary healthcare services, it was recognized that patient populations who have limited proficiency in English or French, and who may be unfamiliar with the healthcare system in general, rely, to a greater extent, on the services provided in hospital emergency departments.

\section{Research findings}

The findings flowing from this research project suggest that a number of health care providers avoid using any interpreters at all, or, at best, call upon the patients' family members and friends to help them overcome the linguistic barrier. However, the findings also show that clinicians in the primary healthcare sector are generally aware of the risks involved in relying on the assistance of family members including children, untrained volunteer interpreters, and staff who speak a second language. At the same time, as we will see below (section 5), the data show that this resistance to systemic change to address this potentially dangerous situation appears to be embedded in both the attitudes and practices of the service providers themselves, and the institutional structures and governance policies within the service delivery systems. 
This wall of resistance has been deemed responsible for much of the difficulty experienced in Canada by immigrant and minority language advocacy groups in trying to ensure community interpreting services for newcomers to Canada and others who have limited proficiency in English and French, including members of Canada's First Nations. The discussion stemming from the findings of this study and one of the recommendations of the project, suggests the opportunity for a paradigm shift within the healthcare sector, and that instead of seeing language barriers simply as a human rights issue, the linguistic barriers within the sector may be addressed within the matrix of the organization's risk management responsibilities.

\subsection{Interpreting quality risks}

Clinical staff were cognizant of a number of the risks encountered when they had to depend on the interpretations provided by family members and friends. They were, for example, aware that although well-meaning, these individuals would provide interpretations that could be tempered by the stress inherent to the role into which they were placed, and the demands of having to cope with and interpret words and concepts which were cognitively challenging. For instance, a speech pathologist assessing a young patient's ability to use action words would show the patient a picture of a child playing with blocks and say, "Describe to me what this child is doing." If the interpreter renders this request as, "Tell me about the child playing with the blocks," then the assessment will be biased and the pathologist's ability to offer treatment will be affected. It should be noted that speech pathologists also work with interpreters in assessing the memory and vocabulary of older LEP/LFP patients after they suffer a stroke (Lewington 2006).

Language, interpreting and other cognitive abilities are perceived to be relatively easy to assess. The prevalence of cases where health care providers call upon people to interpret simply based on their ability to speak two languages attests to that perception. However, in spite of service providers believing that they are able to assess the cognitive skills of their would-be interpreters, they appear to overlook the impact that emotional involvement may have on the quality of interpretation. For example, in depending on family members, friends or other untrained interpreters, health care providers should be aware that an oversight by the health care provider of any emotional ties between a patient's health status and their friends and family members could lead to misinterpretation or to a failure to interpret efficiently. Untrained interpreters may not be able to detach themselves sufficiently from the situation in order to ensure as accurate and complete an interpretation as possible.

As previously indicated, the study revealed that health care providers often depend on family members and friends despite their awareness that the family and friends' emotional relationship to the patient may influence the latter's desire to minimize the patients' or their own pain and discom- 
fort. Respondents noted that, in such cases, interpreters would sometimes answer the questions asked by the health care provider instead of letting the patients speak for themselves.

Some informants who had worked with untrained interpreters spoke about times when they had a sense that problems encountered by these interpreters were not limited to terminology problems. One informant cited the instance of a diabetic patient whose family had trouble coming to terms with the illness. It was later revealed that the patient was not following treatment, and because the health care provider had sensed that there was something unusual going on, that provider called upon the services of a professional interpreter.

\subsection{Financial risks}

One clinical staff member noted that when family members acting as interpreters have a limited knowledge of English themselves, there are situations where after-care instructions are misunderstood, the patient's condition worsens and the patient has to return to the hospital. This tends to support the hypothesis that unprofessional or faulty interpretation may have a direct financial impact on the healthcare system which could be greater than the cost of employing trained, professional interpreters in the first place.

The findings of the literature review suggest that repeat visits to health care providers may add financial pressures to both the already overburdened public healthcare system, and the economy in general. Appointments with physicians often entail time away from the job, and a misdiagnosis or inability to follow through with treatment due to language barriers, could translate into a loss of productivity.

\subsection{Ethical risks}

Because employing the services of an interpreter means that the health care provider will have to involve a third party in the discussion of confidential matters, concerns around breaches of confidentiality were raised time and time again by informants. Informants indicated that this potential risk is considerably heightened when the interpreter is known to the patient. By using family members or friends to interpret, health care providers expose themselves to a potential breach of confidentiality vis-à-vis their patients. Take, for example, a situation where a family member brought in to interpret may have a certain responsibility for, or be affected by, the health problem for which the patient is consulting a doctor. Such would be the case for someone consulting a doctor for an undetected sexually transmitted disease who brings in a relative to interpret, or for a physically molested child whose abuser is also acting as the interpreter.

The issue of confidentiality was also raised in the context of communities of languages of lesser diffusion, where community members are 
likely to have frequent contact with each other. With respect to knowledge related to patients who are diagnosed with stigma-carrying illnesses, ensuring that the patient-doctor consultation is interpreted at all cost is not the lesser evil; it may actually compound an already difficult situation (see Fiola 2004).

Some informants also noted that, because of the possible consequences of misinterpretation in the healthcare sector, children should never be asked to interpret, as they should never have to be responsible for the issues that are at stake.

\subsection{Risks associated with workplace stress}

One informant, who was a bilingual staff member, was called upon to interpret, a role for which she believed she was unqualified. The reservations outlined by the informant included uncertainty about the role to be assumed when helping a colleague to work through the language barrier; the ways in which the lack of formal medical training limits the ability to understand and accurately interpret health care-related topics; and, unfamiliarity with the regionalisms in the language spoken by some patients, which creates difficulty when seeking to interpret idiomatic expressions. Also, one can add that the time that bilingual staff members are taken away from their work to act as interpreters is usually not considered to be part of their normal workload. Thus, others have to cover for them in their absence, or they have to make up themselves for that time away from their usual tasks, which can only lead to added stress in the workplace.

\section{The resistance to working with professional interpreters}

The data suggests that a number of service providers were aware of the liabilities and risks incurred when the delivery of primary healthcare services to patient populations with limited proficiency in English is dependent on the interpretations provided by family, friends, volunteers and bilingual staff, and that the quality of service delivered is compromised in these situations. The research also revealed the presence of systemic hindrances to addressing the linguistic barriers that inhibit delivery of primary healthcare services to patient populations who do not speak either of Canada's official languages.

The areas of resistance addressed in this section relate to the barriers expressed in the attitudes of clinicians, as well as those barriers framed in the policies and practices of the primary healthcare service delivery systems. 


\subsection{Attitudinal resistance}

The research indicates the presence of attitudes that ascribe to the notion that the inability to communicate in English and/or French is the patient's problem. One physician indicated a level of impatience with patients who fail to improve their language skills, in particular elderly patients with poor knowledge of English after living in Canada for many years. Another informant admits to encouraging patients to take ESL classes and is less and less inclined to be linguistically accommodating in the examination room, thus requiring the patients to speak English. A third physician suggested that patients could consult lists of language-congruent doctors, i.e. who practise in languages other than English or French, indicating that this would be a solution to overcome the communication barriers patients are facing. Although this may be part of a solution, it is clear that not all patients have the ability or time to search for language-congruent healthcare professionals. Furthermore, a widespread belief among informants is that if doctors are not explaining the procedures they use to their patients, then the patients should find another physician. In the words of one clinician, "if the fit isn't good, they can go elsewhere."

Finally, one informant was opposed to the idea that the public healthcare system should be responsible for supplying interpreters when Canada has already two official languages, adding that the healthcare system is already financially strained, and the cost of interpretation would only exacerbate the situation. That informant also suggested that patients with limited English should take some responsibility for their own care, which implicitly includes ensuring that they are able to communicate efficiently with their healthcare professionals, either directly or through the services of someone who is able to do it for them.

\subsection{Structural and institutional barriers}

Clinicians in one hospital spoke about the barrier presented by an organizational policy which directed that the interpreters provided by the hospital had to be booked 24 hours in advance of the assignment. In such situations clinicians are directed to call a telephone interpreting service. The informants stated their reluctance to use this service on the grounds that it places a burden on clinicians who have to think of all their questions carefully before they place the call. Indeed, it is difficult for them to get the same interpreter back on the line after the fact if they remember or think of other information they need from the patient. Furthermore, they felt that the flow of conversation between patient and practitioner is stilted and artificial, and, "Practitioners have a difficult time assessing whether the patient has understood. You hand the phone over to the patient and hope that the person on the other end is telling them what you said, you have to have a lot of faith when you use the service." The impersonality of the communication and the risk that some information will be missed were also of concern. Clinicians 
in these situations indicated that they had no alternative but to rely on whoever was available in crisis situations to assist them in their communication with clients with whom they did not have a language in common.

Other informants discussed financial barriers which affected the ability of family practice physicians to contract with appropriately trained spoken language interpreters. The provincial governments, who sponsor healthcare insurance plans in every Canadian province, usually do not provide for the billing of interpreter services, except to French-speaking minority communities outside Quebec and to English-speaking minority communities in Quebec, or for the additional billable ${ }^{2}$ time needed when services are provided with the assistance of an interpreter. The research also revealed that even in situations where budgets can cover the costs of trained interpreters, there was one incident where the manager decided that the funding would be better spent elsewhere and directed that service providers rely on the services of bilingual staff members, volunteer interpreters or family members for their interpretation needs.

While the three-city research revealed the presence of a spectrum of formal and ad hoc responses to addressing the linguistic barriers in the delivery of primary healthcare services, evidence shows that there is limited integration of the provision of trained community interpreter services into the primary healthcare sector, inadequate if not non-existent policy development and an uneven understanding among clinicians of the risks associated when working with untrained interpreters in the provision of services to patient populations with limited official language proficiency.

Some of the data collected in this three-city research were consistent with findings published in Bowen (2001). Bowen also signalled that language, rather than cultural beliefs and practices of patients, may be the most significant barrier to initial contact with healthcare services, and, while the evidence is limited, there is some suggestion that, in Canada, limited proficiency in English and French may result in high utilization of specialists and diagnostic services. In Bowen, evidence also demonstrated that these populations have reduced access to mental health and counselling-related services. In addition, Bowen suggests that delays in seeking care, lack of understanding of diagnoses, and the inability to comply with treatment measures may also be the consequences of language barriers.

Of particular concern were the ways in which language barriers may compromise the quality of care as a result of the failure to obtain patients' informed consent.

\section{The paradigm shift}

In some countries, language access advocacy groups have had success in the past in convincing decision-makers that public services can only be truly public if they are accessible to the whole of the public, regardless of their physical abilities, gender, religious beliefs, race and language background. Canada has made considerable progress in this direction, but it still falls 
short of having a national policy on addressing language barriers to public services, including healthcare services. This section explains how it was, and is often still, believed that human rights legislation may prove to be the way to ensure full language access, and how other avenues may be explored to that end, especially that of risk management.

\subsection{Language access as a human rights issue}

An example of human rights legislation used as a driving force behind Canadian public policy can be found in the case of sign language interpreting in the healthcare system. The Canadian Human Rights Act identifies disability as a ground for discrimination and individuals requiring sign language interpreters are covered by the Act. All provinces and territories have similar laws forbidding discrimination in their areas of jurisdiction, which includes health. The 1997 Eldridge Decision by the Supreme Court of Canada, which stated that the failure to provide sign language interpretation where it is needed for effective communication in the delivery of healthcare services violates the rights of deaf people, reinforced this entitlement. The Eldridge Decision states that governments cannot escape their constitutional obligations to provide equal access to public services. There is no equivalent ruling for spoken language interpreting, as the inability to speak one language but not another is not a physical handicap ${ }^{3}$. There are few public records of the impact of miscommunication due to spoken language barriers on the lives of those who have limited proficiency in English or French.

These and other issues were highlighted at a National Symposium ${ }^{4}$ which was constituted to disseminate the findings of the research project, and begin the process of identifying viable strategies for strengthening the access to primary health care by those patient populations who have limited proficiency in Canada's official languages.

In addressing the Symposium, Raj Anand, former Ontario Human Rights Commissioner, talked about the inequities facing members of the Canadian population who are unable to communicate in French or English. Anand concluded that while the Eldridge Decision recognizes that effective communication is essential to the provision of medical services, it cannot be directly applied to the broader context of those immigrants, minority Francophone, Anglophone or First Nations communities who have limited proficiency in Canada's official languages.

\subsection{Language access as a risk management issue}

At the same symposium, however, a second presentation (Nielsen 2005) outlined the beginning of a systemic response to addressing language barriers in the healthcare sector. This argument posited that without an overriding legal framework to guide programs, negative responses to language barriers have continued to grow. It further suggested that to maintain positive 
momentum and ensure relevance to the ever-changing state of health care in Canada it is essential to re-examine approaches to, and perspectives on, language-access services. ${ }^{5}$

The presentation challenged the audience with finding ways for ensuring that the evidence gathered in the research project could be integrated into organizational planning and program development, and demonstrated the way in which a paradigm shift to a risk management approach might be a pathway for change.

It was suggested that rather than viewing interpreter services as a new, add-on program for patients, addressing language barriers must be understood as part of an integrated strategy for achieving organizational objectives. This shift focuses not on individual and community deficits (lack of fluency in an official language), but on organizational deficits (failure of healthcare organizations to ensure effective and equitable service provision in a culturally diverse society). Integrating this shift involves changing the language we use and "interpreting" the evidence between decision-makers and the community. Credible evidence needs to be included in the decisionmaking process and knowledge-brokering roles may help foster effective communication of the evidence to decision-makers and clinicians. In order to remain viable and relevant, language services need to be aligned with current healthcare agendas, including those of quality, patient safety and risk management. Inclusion in these agendas will ensure that language programs are not viewed as an expensive add-on service, but rather as an integral part of the overall framework aimed at meeting organizational goals. The move away from "cultural sensitivity" towards service delivery and service outcomes will frame language access as a clinical issue, with clear effects on both patients/clients and an organization's bottom line.

From a logistical point of view, it was demonstrated how policy change must be the vehicle to mandate language-access services. Four stages of policy and planning need to be considered:

- $\quad$ Agenda Setting - Attain recognition among decision-makers that language access requires public or organizational resources and effort;

- Formulation - Generate a solution to language access and a strategy to address it;

- $\quad$ Implementation - Put the strategy into practice;

- Evaluation - A strategic means to integrate incrementally interpreter services into the organization's operations (Nielsen 2005).

A response ${ }^{6}$ to the challenge is currently being developed in Vancouver, British Columbia. This response is contextualized within the findings of the three-city study, which, as we have seen, demonstrated the lack of understanding among healthcare personnel of the need for definitive and clear policies mandating the use of language services. It also revealed that personnel providing and regulating healthcare services are unaware of the profound implications and risks associated with linguistically inaccessible and culturally dissonant services. 
The main goal of this initiative is to develop, test, evaluate and ultimately implement an Assessment for Risk Management (ARM) tool to assess healthcare access for non- and limited-English speaking populations. Specifically, this approach to risk management will be developed based on the Canadian Council of Health Services Accreditation (CCHSA) standards ${ }^{7}$. The objectives of the project are (1) to develop a risk/control matrix based on the CCHSA standards; (2) to create a practical application for the ARM tool which is aimed at highlighting issues of access and appropriateness around language concerns. Specifically, the tool will be used to assess the health organization's capacity to manage risks related to language incongruity between health care providers, or the system they work in, and healthcare clients. Each question of the ARM Tool will focus on critical points marking a clinical pathway where a risk-control practice standard should or could be employed. Once developed, the tool will be pilot-tested at two sites in British Columbia, i.e. the Renal Program (Fraser Health Authority) and the British Columbia Cancer Centre (Provincial Health Services Authority).

When tested, evaluated and, if required, modified, the ARM tool will be transferable to other healthcare sites. It can be expected that this tool will help demonstrate and substantiate the claims that eliminating language barriers in the healthcare system leads to the improvement of the accessibility and quality of health care to patients who cannot or do not yet speak either of Canada's official languages.

\section{Conclusion}

As we have seen, the systemic integration of trained community interpreters into the healthcare system still has a long way to go. As we have also seen, the structural and institutional resistance, as well as attitudinal barriers to this movement, is embedded in policies and regulations in the public and institutional sectors, as well as in the attitudes of those service providers who are ill-prepared to consider the effects of miscommunication on the quality of health care delivered to those patients who have a limited proficiency in Canada's official languages. Implicit in the paradigm shift outlined in this article - the movement from needs assessment to risk management - is the perception that the healthcare system in Canada is becoming increasingly aware of the liabilities inherent in the delivery of services which put at risk the diagnosis and treatment of patient populations. This perception is evident in the attention being paid to 'risk management' at the institutional level. The development of the Assessment for Risk Management tool outlined above is, as far as we are aware, the first time that specific attention is being paid to the development of an organizational assessment mechanism which pays particular attention to the needs of those patient populations from minority language backgrounds. It has the potential to radically change the attitudes of those responsible for the delivery of healthcare services, and the quality of care for those who have limited pro- 
ficiency in Canada's official languages. In many respects, this paradigm shift suggests that the provision of language services and the elimination of language barriers must be understood as part of an integrated strategy for achieving organizational and social development objectives.

Initiatives in other parts of the world have seen the integration of language barriers within the general framework of hospital risk management portfolios. However, considering language barriers from a risk-management perspective instead of a human-rights position indicates a paradigm shift in Canada, and possibly elsewhere. Successful testing of this tool could lead to the beginning of its implementation within the healthcare system in this country.

The emergent method of research we used and which led to the development of the ARM tool, as well as the research results obtained by Bowen (2001) enabled us to uncover a number of potential risks embedded in the inabilities of health care providers to communicate effectively with LEP/LFP patient populations. It is hoped that the successful testing of the ARM tool and a wider diffusion of the results and methodology used in developing that tool will result in other institutions seeing the benefits of addressing the problem posed by language barriers in providing quality of care to language minorities.

\section{Bibliography}

Abraham, Diana \& Kristofer Nielsen (2004). "Healthcare Interpreter Services: Strengthening Access To Primary Healthcare. Preliminary Research Findings". Toronto [unpublished paper $]^{8}$.

Bowen, Sarah (2001). "Language Barriers in Access to Healthcare". Ottawa: Health Canada. On line at: www.hc-sc.gc.ca/hcs-sss/pubs/care-soins/2001-lang-acces/index_e.html (consulted 18.03.2006).

Canadian Council on Health Services Accreditation (2005). “A Look Inside Canada's Healthcare System. Canadian Health Accreditation Report 2004". On line at: http://www.cchsaccass.ca/pdf/2004report.pdf (consulted 30.03.2006).

Fiola, Marco A. (2004). "Le 'gain et le dommage' de l'interprétation en milieu social". TTR, 17:2, p. $115-130$.

Government of Canada (1976-1977). "Canadian Human Rights Act”. Ottawa: Justice Canada. On line at http://laws.justice.gc.ca/en/H-6/243963.html (consulted on 30.03.2006).

Hemlin, Isabelle \& Heather Clarke (2006). Health Care Interpreter Services: Strengthening Access to Primary Health Care Montreal Regional Report: An overview of the history, development and current state of interpreter services in Quebec. Montreal, Quebec: Agence de la santé et des services sociaux de Montréal. Online at: http://www.accessalliance.ca/media/SAPHCMontrealRegionalReport.pdf (consulted 31.08.2006)

Hoen, Beth, Kristofer Nielsen, Axelle Janczur, Diana Abraham \& Marco Fiola (2006). Health Care Interpreter Services: Strengthening Access to Primary Health Care, Toronto Regional

Report: An overview of the history, development and current state of interpreter services in Ontario. Toronto: The Healthcare Interpretation Network. Online at: http://www.accessalliance.ca/media/SAPHCTorontoRegionalReport.pdf (consulted 31.08.2006).

Hoen, Beth, Kristofer Nielsen \& Angela Sasso (2006). Health Care Interpreter Services: Strengthening Access to Primary Health Care National Report: An overview of the accomplishments, outcomes and learnings of the SAPHC project. Toronto: The Healthcare Interpretation Network. Online at: http://www.accessalliance.ca (consulted 31.08.2006)

Lewington, Jennifer (2006). "Bridging the language gap". The Globe and Mail. Toronto, p. A11, June 26. 
Nielsen, Kristofer (2005). Primary Healthcare for All: Overcoming Linguistic Barriers. A synthesis of presentations and emerging themes from the Healthcare Interpreter Services: Strengthening Access to Primary Healthcare (SAPHC) Project's National Symposium. May 4-6, 2005, Orangeville, Ontario [unpublished monograph].

Provincial Language Services (2005). Walk Your Shop: Assessment for Risk Management (ARM) Tool on Healthcare Access for Non- and Limited-English Speaking Populations. Pilot Project Proposal. Vancouver [unpublished pilot project proposal].

Sasso, Angela, \& LaPel Consulting and Design (2005). Strengthening Access to Primary Healthcare Project - Vancouver Regional Report. Online at: www.accessalliance.ca (consulted 31.08.2006).

Sasso, Angela (2006). Health Care Interpreter Services: Strengthening Access to Primary Health Care Vancouver Regional Report: An overview of the history, development and current state of interpreter services in British Columbia. Vancouver Provincial Languages Services. Online at: http://www.accessalliance.ca (consulted 31.08.2006).

Shahsiah, Sara \& Hélène Grégoire (2006). Health Care Interpreter Services: A literature review. Health Care Interpreter Services - Strengthening Access to Primary Care. Toronto: The Healthcare Interpretation Network. Online at: http://www.accessalliance.ca (consulted 31.08.2006)

Supreme Court of Canada (1997). "Eldridge v. British Columbia (Attorney General)". On line at: http://www.culturalrenewal.ca/lex/lex-14.htm (consulted 30.03.2006).

1 For the purpose of this research primary healthcare services were limited to those delivered by physicians and nurses in family practice, public health nurses, social workers in a cross-section of healthcare locations, counsellors in settlement agencies and physicians and nurses in hospital emergency departments.

2 Time for which a physician is entitled to claim payment from the public health system. There are provisions dealing with extra time physicians spend in order to accommodate patients with physical disabilities, but not with patients with whom they do not share a language.

However, whether it could be recognized as discrimination on the ground of race or national or ethnic origin is still to be demonstrated.

4 Primary Health Care for All: Overcoming the Linguistic Barrier, Orangeville, Ontario, Canada, May 4-6, 2005.

5 Presentation delivered by Sarah Bowen, Director of Research and Evaluation with the Winnipeg Regional Health Authority, and Elizabeth Stanger, the Regional Coordinator of Language Services, Cross-cultural Health and Diversity with Vancouver Coastal Health.

6 This response is a pilot project aimed at testing a risk assessment tool adapted to measure the risks related to the provision of services that are not linguistically adapted to non-English speakers.

7 Standards cover areas applicable to all organizations: governance and management, environmental management, human resources, and information management. They also address client services across a diverse range of health sectors, from home care to mental health to health systems. Each standard has a set of criteria that describes activities or processes required to meet the standard (CHSSA 2004: 7).

8 All unpublished papers and documents referred to in this bibliography included preliminary findings, which were then used to develop the final documents available online. They can be obtained through the Health Interpretation Network. 